\title{
Mathematical Analysis of Financial Model on Market Price with Stochastic Volatility
}

\author{
Mitun Kumar Mondal', Md. Abdul Alim², Md. Faizur Rahman³, Md. Haider Ali Biswas' \\ ${ }^{1}$ Mathematics Discipline, Science Engineering and Technology School, Khulna University, Khulna, Bangladesh \\ ${ }^{2}$ Department of Mathematics, University of Chittagong, Chittagong, Bangladesh \\ ${ }^{3}$ South East Engineering College, University of Rajshahi, Rajshahi, Bangladesh \\ Email:mkmondal.bd@gmail.com, alim_math@yahoo.com, sbit.khulna@yahoo.com,mhabiswas@yahoo.com
}

How to cite this paper: Mondal, M.K., Alim, M.A., Rahman, M.F. and Biswas, M.H.A. (2017) Mathematical Analysis of Financial Model on Market Price with Stochastic Volatility. Journal of Mathematical Finance, 7, 351-365.

https://doi.org/10.4236/jmf.2017.72019

Received: October 21, 2016

Accepted: May 15, 2017

Published: May 19, 2017

Copyright (C) 2017 by authors and Scientific Research Publishing Inc. This work is licensed under the Creative Commons Attribution International License (CC BY 4.0).

http://creativecommons.org/licenses/by/4.0/

\begin{abstract}
The Heston model is one of the most popular stochastic volatility models for option pricing to measure the volatility of different parameters in the financial market. In this work, we study the statistical analysis of Heston Model by partial differential equations. The model proposed by Heston takes into account non-lognormal distribution of the assets returns, leverage effect and the important mean reverting property of volatility. We have assayed on the return distribution on the basis of different values of correlation parameter and volatility, then we measure the effects of parameters $\rho$ (correlation coefficient) and $\sigma$ (standard deviation) for different situation such as $\rho>0, \sigma>0, \rho=0, \sigma=0, \rho$ $<0, \sigma<0$ etc. On return distribution of Heston Model which indicates market condition for buyers and sellers to buy and sell options. All solvers used in this analysis are implemented using MATLAB codes and the simulation results are presented graphically.
\end{abstract}

\section{Keywords}

Stochastic Volatility, Black Scholes Biases, Heston Model, Black-Scholes Equation, Calibration, Characteristic Functions

\section{Introduction}

In 1970, Fischer Black, Myron Scholes and Robert Merton derived the "BlackScholes model" (sometimes known as "Black-Scholes-Merton") which changed the way and impact the world of pricing derivatives using stocks as the underlying asset [1] [2]. For this great contribution to study and analyze the financial market, Scholes and Merton were awarded the Nobel Prize in economics in 1997 (Fischer Black died in 1995). It is then easy to understand the significant impact and necessity of this formula in studying the market price in the economics and 
finance. Since then many researchers have been aptly used to describe Black and Scholes' model to option pricing theory. Despite subsequent development of option theory, the original Black-Scholes (BS) formula for a European call option remains the most successful and widely used application. This formula is particularly useful because it relates the distribution of spot returns to the cross-sectional properties of option prices.

Another great obtainment in the financial market is Heston's stochastic volatility model, which helps to resolve a shortcoming of the BS model. More precisely, we can say that models based on BS assume that the underlying volatility is constant over the life of the derivative and unaffected by the changes in the price level of the underlying security. Howsoever, these models cannot explain long-observed features of the implied volatility surface like as volatility smile and skew, which indicate that implied volatility does tend to vary with respect to strike price and expiry. By assuming that the volatility of the underlying price is a stochastic process rather than a constant, it becomes possible to model derivatives more accurately.

Now-a-days, Heston model is considered as one of the most popular stochastic volatility option pricing models, which is motivated by the widespread evidence that volatility is stochastic and that the distribution of risky asset returns has tail (s) longer than that of a normal distribution [3]. A Stochastic volatility model which has correlation of price and volatility innovations can address both empirical stylized facts. The Stochastic volatility option pricing model was developed by the contributions of many researchers. Among them, Johnson and Shanno [4] showed the option pricing for the changing of variance, Wiggins [5] presented a numerical solution of the call option valuation problem given a fairly general continuous stochastic process for return volatility, Hull and White gave an analysis of the bias in option pricing caused by a stochastic volatility in [6] and the pricing of options on assets with stochastic volatilities in [7]. Scott [8] developed an option pricing model when the variance changes randomly. Stein and Stein studied the stock price distributions that arise when prices follow a diffusion process with a stochastically varying volatility parameter in [9] and Heston showed a closed solution for the options with stochastic volatility in [3]. Also a semi-closed form solution was derived based on characteristic function of the price distribution [3]. All of the authors used a mathematical model to explain the option pricing model because of mathematical modeling can play a significant role to discuss different types of real phenomena which lead to design better prediction. As a result many authors use mathematical modeling in different spheres. For example, Biswas et al. [10] studied the potential impacts of Global Climate Change in Bangladesh. Mondal and Biswas [11] developed a mathematical model to describe the transmission of Nipah virus between bats and human. Neilan and Lenhart [12] showed the application optimal control strategy in disease modelling. Biswas and Haque [13] discussed the necessity of nonlinear dynamical system to control the infectious disease. We also refer readers to [1] [14] [15] [16] and the references within for the study of more ap- 
plications of mathematical modeling in real life problems.

The real issue was whether the ideal behavior was defensible in the derivation of this formula, Heston made several simplifying assumptions [3]. The theoretical models may tell us, ultimately the prices of financial instruments such as options are determined by the market. One of the key pricing parameters in the Heston model is the volatility or the expected standard deviation of the underlying assets assuming that asset price follows a geometric Brownian motion with constant volatility consequently the options on the same asset, but with different strike prices and maturity dates. Since this number varies across different options with the same underlying, and when plotted on a graph against the strike price of these options formed smile characteristic. But this does not appear acceptable as the volatility should only depend on the underlying asset. This event suggests that the original Black-Scholes model is not structurally able to accurately describe option prices and their dynamics. Prior solution can be categorized in two situations where the assumption made by Black-Scholes that volatility be constant had been relaxed in both cases. Heston models add an additional source of randomness to the model. On the other hand, local volatility models make use of a surface which describes the volatility over different maturities and underlying asset prices. Only key solution of local volatility is to determine local volatility function by calibration observed market prices. The famous Heston model has been intensively studied and used as the foundation for almost any option pricing formula in today's financial markets. But we introduced an interpolators approach called finite difference. Finite difference is a function of the distance of the point to the origins which are particularly useful in interpolating data and are widely used in both pattern recognition and surface investigation. This investigation helps investor to know price options more accurately. It focuses on the reconstruction of unknown functions from known data by scientific way. Generally finite difference method is multivariate and they may be solutions of partial differential equations satisfying certain additional conditions. However, the reconstruction of multivariate functions from data can only be done if the space furnishing the trial functions and makes function sets as interpolators. Finite difference methods also provide interpolation of function values given at irregularly positioned points for any value of observation index.

The main goal of this paper is to measure true volatility and experimental volatility as well as compare them to examine the present situation of the share market. We want to measure the effect of different parameters of Heston model on return distribution and effect on implied volatility surface with strike and maturity as well as discuss the pricing through the Partial Differential Equation. This work is actually a statistical analysis of option price Heston model and a calculation of different error measurement to test the consistency of different functions.

\section{Black-Scholes Equation}

Black and Scholes [17] first proposed this equation bringing a huge change in 
the financial market, and it was the first time when people knew how to make a price for an option. The model was developed under the following assumptions:

A1. The stock price follows the stochastic process $d s=\mu S d t+\sigma S d W$, with fixed $\mu$ and $\sigma$;

A2. Unrestricted short-selling of stock, with full use of short-sale proceeds;

A3. No transactions costs and taxes;

A4. No dividends are paid during the life of the option;

A5. There are no riskless arbitrage opportunities:

A6. It is based on European options;

A7. The risk-free rate of interest $r$ is constant and same for all maturities;

A8. Continuous trading;

In order to make a price for a call option on a non-dividend paying stock with the BS Equation, we need to know current stock price, strike price, risk-free interest rate, volatility and time to maturity. It is easy to get all above inputs variables in the market except the volatility. For the price of a non-dividend paying call option, the BS equation is described as:

$$
C(S, t)=S N\left(d_{1}\right)-K \mathrm{e}^{-r(T-t)} N\left(d_{2}\right)
$$

where, $d_{1}=\frac{\ln \left(\frac{s}{k}\right)+\left(r+\frac{\sigma^{2}}{2}\right)(T-t)}{\sigma \sqrt{(T-t)}}$ and $d_{2}=d_{1}-\sigma \sqrt{(T-t)}$

Here, $S$ is the stock price at time $t, T$ is the maturity date, $K$ is the strike price, $N\left(d_{2}\right)$ is the cumulative normal distribution, $\sigma$ is the volatility. Although Black-Scholes equation is still widespread used in the market, much evidence has shown that the assumption of fixed volatility is not suitable for actual data. Consequently, in this dissertation, we consider the volatility following a stochastic process rather than a constant during the life of a call option.

\section{The Heston Stochastic Volatility Model}

The crude assumption of constant volatility in the Black-Scholes formula causes problem. One model where the volatility is a stochastic process is the Heston Stochastic Volatility Model [3], which is an extended version of the BlackScholes stochastic differential equations (SDE) with a volatility. The Heston Model takes the non-log normal distribution of the assets returns and the leverage effect into account, the correlation between the two Wiener processes. The Heston Model defined by following stochastic processes:

$$
\begin{aligned}
& d S_{t}=\mu S_{t} d t+\sqrt{V_{t}} S_{t} d W_{t}^{1} \\
& d V_{t}=\kappa\left(\theta-V_{t}\right) d t+\sigma \sqrt{V_{t}} d W_{t}^{2}
\end{aligned}
$$

and where $W_{t}^{1}$ and $W_{t}^{2}$ are correlated Wiener processes with $\rho$, i.e.

$$
d W_{t}^{1} d W_{t}^{2}=\rho d t
$$

where, $\mu$ is the drift coefficient of the stock price, $\theta$ is the long term mean of variance, $\kappa$ is the rate of mean reversion, $\sigma$ is the volatility of volatility, $s_{t}$ 
and $v_{t}$ are the price and volatility process respectively, where $\left\{v_{t}\right\}_{t \geq 0}$ is a square root mean reverting process, first used by with long run mean $\theta$, and rate of reversion $\kappa$. To take into account the leverage effect, stock returns and implied volatility are negatively correlated, $w_{t}^{1}$ and $w_{t}^{2}$ are correlated wiener process and the correlation coefficients is $\rho$. All the parameters $\mu, \kappa, \theta, \sigma, \rho$ are the time and state homogeneous.

\section{Analysis of Heston Model}

\subsection{Partial Differential Equation Approach of Heston Model}

For some stochastic volatility models, one can find a partial differential equation (PDE), the value of any option must be satisfied by such a PDE. For Heston's Stochastic Volatility model, a PDE exists, but calculation is quite complicated due to the difficult estimation of the market price of volatility risk. In order to price options in the SV model, we can apply no-arbitrage arguments, or use the risk-neutral valuation method. First we discuss the no-arbitrage method. The riskless portfolio is constructed as in the Black-Scholes model. But the construction method is different. In the SV option pricing model, there is only one traded risky asset $S$ but two random sources $d W_{t}^{1}$ and $d W_{t}^{2}$. So the market is incomplete. We cannot perfectly replicate the option solely with the underlying stock. No-arbitrage arguments are not enough to give the option price. We need additional assumptions. In the following derivation, equilibrium arguments are also employed. We know that the market can be completed by adding any option written on stock $S$. Simply, the market is complete when we have two traded assets, the underlying asset $S$ and a benchmark option $V_{1}$. Then all other options can be replicated by these two traded assets.

To proceed, consider a self-financing/risk-less portfolio with value $\Pi$ consisting of an option with value $V(S, v, t)$ which we want to price, $-\Delta$ units of the underlying asset $S$ and, in order to hedge the risk associated with the random volatility, $-\Delta_{1}$ units of benchmark option with value $V_{1}(S, v, t)$.

Hence,

$$
\Pi=V-\Delta S-\Delta_{1} V_{1}
$$

The portfolio is self-financing i.e. for risk-less portfolio, so that

$$
d \Pi=d V-\Delta d S-\Delta_{1} d V_{1}
$$

By applying two dimensional form of Ito's formula, we have

$$
\begin{aligned}
d \Pi & =\left\{\frac{\partial V}{\partial t}+\frac{1}{2} v S^{2} \frac{\partial^{2} V}{\partial S^{2}}+\rho \sigma S v \frac{\partial^{2} V}{\partial S \partial v}+\frac{1}{2} v \sigma^{2} \frac{\partial^{2} V}{\partial v^{2}}\right\} d t+\frac{\partial V}{\partial S} d S+\frac{\partial V}{\partial v} d v \\
& -\Delta_{1}\left\{\frac{\partial V_{1}}{\partial t}+\frac{1}{2} v S^{2} \frac{\partial^{2} V_{1}}{\partial S^{2}}+\rho \sigma S v \frac{\partial^{2} V_{1}}{\partial S \partial v}+\frac{1}{2} v \sigma^{2} \frac{\partial^{2} V_{1}}{\partial v^{2}}\right\}-\Delta_{1} \frac{\partial V_{1}}{\partial S} d S \\
& -\Delta_{1} \frac{\partial V_{1}}{\partial v} d v-\Delta d S
\end{aligned}
$$

Now, we can rewrite it by collecting the terms of $d S$, $d t$ and $d v$ 


$$
\begin{aligned}
d \Pi & =\left\{\frac{\partial V}{\partial t}+\frac{1}{2} v S^{2} \frac{\partial^{2} V}{\partial S^{2}}+\rho \sigma S v \frac{\partial^{2} V}{\partial S \partial v}+\frac{1}{2} v \sigma^{2} \frac{\partial^{2} V}{\partial v^{2}}\right\} d t+\left(\frac{\partial V}{\partial v}-\Delta_{1} \frac{\partial V_{1}}{\partial v}\right) d v \\
& -\Delta_{1}\left\{\frac{\partial V_{1}}{\partial t}+\frac{1}{2} v S^{2} \frac{\partial^{2} V_{1}}{\partial S^{2}}+\rho \sigma S v \frac{\partial^{2} V_{1}}{\partial S \partial v}+\frac{1}{2} v \sigma^{2} \frac{\partial^{2} V_{1}}{\partial v^{2}}\right\} d t \\
& +\left(\frac{\partial V}{\partial S}-\Delta_{1} \frac{\partial V_{1}}{\partial S}-\Delta\right) d S
\end{aligned}
$$

To make the portfolio riskless, we choose

$$
\begin{aligned}
& \frac{\partial V}{\partial v}-\Delta_{1} \frac{\partial V_{1}}{\partial v}=0 \\
& \frac{\partial V}{\partial S}-\Delta_{1} \frac{\partial V_{1}}{\partial S}-\Delta=0
\end{aligned}
$$

To eliminate the terms $d S$ and $d v$, we solve the Equation (8) and (9) as

$$
\begin{aligned}
& \Delta_{1}=\frac{\frac{\partial V}{\partial v}}{\frac{\partial V_{1}}{\partial v}} \\
& \Delta=\frac{\partial V}{\partial S}-\Delta_{1} \frac{\partial V_{1}}{\partial S}=\frac{\partial V}{\partial S}-\frac{\frac{\partial V}{\partial V}}{\frac{\partial V_{1}}{\partial v}} \frac{\partial V_{1}}{\partial S}
\end{aligned}
$$

The portfolio is risk free if we rebalance the Equation (7) according to (10) and (11). On the other hand, the riskless portfolio must earn a risk free rate, i.e. the return of this risk-free portfolio must equal the (deterministic) risk-free rate of return. Otherwise, there would be an arbitrage opportunity.

$$
\begin{aligned}
d \Pi & =\left\{\frac{\partial V}{\partial t}+\frac{1}{2} v S^{2} \frac{\partial^{2} V}{\partial S^{2}}+\rho \sigma S v \frac{\partial^{2} V}{\partial S \partial v}+\frac{1}{2} v \sigma^{2} \frac{\partial^{2} V}{\partial v^{2}}\right\} d t \\
& -\Delta_{1}\left\{\frac{\partial V_{1}}{\partial t}+\frac{1}{2} v S^{2} \frac{\partial^{2} V_{1}}{\partial S^{2}}+\rho \sigma S v \frac{\partial^{2} V_{1}}{\partial S \partial v}+\frac{1}{2} v \sigma^{2} \frac{\partial^{2} V_{1}}{\partial v^{2}}\right\} d t
\end{aligned}
$$

$=r \prod d t=r\left(V-\Delta S-\Delta_{1} V_{1}\right) d t \quad$ (by using Equation (7))

$$
\therefore d \Pi=r\left(V-\Delta S-\Delta_{1} V_{1}\right) d t
$$

Using above two equations we have,

$$
\begin{aligned}
& \frac{\frac{\partial V}{\partial t}+\frac{1}{2} v S^{2} \frac{\partial^{2} V}{\partial S^{2}}+\rho \sigma S v \frac{\partial^{2} V}{\partial S \partial v}+\frac{1}{2} v \sigma^{2} \frac{\partial^{2} V}{\partial v^{2}}+r S \frac{\partial V}{\partial S}-r V}{\frac{\partial V}{\partial v}} \\
& =\frac{\frac{\partial V_{1}}{\partial t}+\frac{1}{2} v S^{2} \frac{\partial^{2} V_{1}}{\partial S^{2}}+\rho \sigma S v \frac{\partial^{2} V_{1}}{\partial S \partial v}+\frac{1}{2} v \sigma^{2} \frac{\partial^{2} V_{1}}{\partial v^{2}}+r S \frac{\partial V_{1}}{\partial S}-r V_{1}}{\frac{\partial V_{1}}{\partial v}}
\end{aligned}
$$

Notice that the left-hand side is a function of $V$ only and the right-hand side is a function of $V_{1}$ only. The only way that this equation holds is that both sides are 
equal to some function, i.e. $g$ only depends on the independent variables $S, v$ and $t$. Setting $g=(\kappa(\theta-V)-\Lambda(S, v, t) \sigma \sqrt{V})$ yields a special case of a socalled affine diffusion process. For this class of processes, the pricing PDE is tractable analytically. In this case we have,

$$
\begin{aligned}
& \frac{\partial V}{\partial t}+\frac{1}{2} v S^{2} \frac{\partial^{2} V}{\partial S^{2}}+\rho \sigma S v \frac{\partial^{2} V}{\partial S \partial v}+\frac{1}{2} v \sigma^{2} \frac{\partial^{2} V}{\partial v^{2}}+r S \frac{\partial V}{\partial S}-r V \\
& =(\kappa(\theta-V)-\Lambda(S, v, t) \sigma \sqrt{V}) \frac{\partial V}{\partial v}
\end{aligned}
$$

Now replacing $V$ by $U(S, V, t, T)$ and $v$ by $V$, we have

$$
\begin{aligned}
& \frac{1}{2} V S^{2} \frac{\partial^{2} U}{\partial S^{2}}+\rho \sigma S V \frac{\partial^{2} U}{\partial S \partial V}+\frac{1}{2} V \sigma^{2} \frac{\partial^{2} U}{\partial V^{2}}+r S \frac{\partial U}{\partial S}-r U \\
& +\frac{\partial U}{\partial t}+(\kappa(\theta-V)-\Lambda(S, v, t) \sigma \sqrt{V}) \frac{\partial U}{\partial V}=0
\end{aligned}
$$

$\Lambda(S, v, t)$ is called the market price of volatility risk. According to Heston's assumption, the market price of volatility risk is proportional to volatility i.e. to the square root of the variance.

$$
\Lambda(S, v, t) \alpha \sqrt{V} \Rightarrow \Lambda(S, v, t)=k \sqrt{V},
$$

where $k$ is the proportional constant.

Multiplying both sides of Equation (16) by $\sigma \sqrt{V}$, then we have,

$$
\Lambda(S, v, t) \sigma \sqrt{V}=k \sigma V
$$

Thus Equation (16) becomes,

$$
\begin{aligned}
& \frac{1}{2} V S^{2} \frac{\partial^{2} U}{\partial S^{2}}+\rho \sigma S V \frac{\partial^{2} U}{\partial S \partial V}+\frac{1}{2} V \sigma^{2} \frac{\partial^{2} U}{\partial V^{2}}+r S \frac{\partial U}{\partial S}-r U \\
& +\frac{\partial U}{\partial t}+(\kappa(\theta-V)-\lambda V) \frac{\partial U}{\partial V}=0
\end{aligned}
$$

Therefore $\lambda(S, V, t)$ represents the market price of volatility risk. The price of volatility risk $\lambda(S, V, t)$ is independent of particular asset. It can be obtained theoretically from any asset depending on volatility risk. Assume the strike price to be $K$ and expiring time $T$. The price is considered in rectangular area of $[0, \infty] \times[0, \infty]$ and on horizontal time $[0, T]$. Then For European call option the option price obeys Equation (4.14) with boundary,

$$
\begin{aligned}
& U(S, v, t)=\max (0, S-k) \\
& U(0, v, t)=0 \\
& \frac{\partial U}{\partial S}(\infty, v, t)=1 \\
& r S \frac{\partial U}{\partial S}(S, 0, t)+\kappa \theta \frac{\partial U}{\partial V}(S, 0, t)-r U(S, 0, t)+\frac{\partial U}{\partial t}(S, 0, t)=0 \\
& U(S, \infty, t)=S
\end{aligned}
$$

This choice of market price of volatility risk gives us analytical advantages. The drift term of the specified process (4) is an affine function of the state variable itself. The affinity makes the model easier to solve. Since the diffusion of the 
variance process is also proportional to the square root of the variance, the product of the market price of risk and the diffusion is proportional to variance itself. As a result, the drift term will remain affine under the Equivalent Martingale Measure (EMM). This particular market price of volatility risk helps the model to have a closed-form solution. We can also apply the risk-neutral valuation method to the SV model. The market is incomplete. But it is still free of arbitrage. The equivalent martingale measure is not unique. We have to choose one of all these measures to price the options. So the price of the option is also not unique. It will depend on which equivalent martingale measure we use.

\subsection{The Effect of Parameters on Return Distribution}

There are many economic, empirical, and mathematical reasons for choosing a model with such a form for a detailed statistical/ empirical analysis).Empirical studies have shown that an asset's log-return distribution is non-Gaussian. It is characterized by heavy tails and high peaks (leptokurtic). There is also empirical evidence and economic arguments that suggest that equity returns and implied volatility are negatively correlated (also termed 'the leverage effect'). This departure from normality plagues the Black-Scholes-Merton model with many problems. In this work, we will show effect of effects of $\rho$ and $\sigma$ on return distribution.

\subsection{Effects of $\rho$ on Return Distribution}

Consider that $\rho$ denotes the correlating factor between the sources of randomness for the underlying and the volatility. $\rho$ can be interpreted as the correlation between the returns and the volatility of the asset. Therefore it captures the leverage effect, affecting the heaviness of the tails, thus the skewness of the return distribution. Intuitively, if $\rho<0$, then volatility will increase as the asset price return decreases, this will spread the left tail and squeeze the right tail of the distribution creating a fat left-tailed distribution. Conversely, if $\rho>0$, then volatility will increase as the asset price/return increases. This will spread the right tail and squeeze the left tail of the distribution creating a fat right-tailed distribution and if $\rho=0$ the skewness is close to zero. As a result $\rho$, affects the skewness of the distribution. Figures 1-5 show the effect $\rho$ for different values. However, Figures 4-12 are similar to those presented in [18], but in this study we have investigated the behaviors of the volatility by changing the values of the parameter $\rho$ and $\sigma$. The simulation results have been shown in Figures 4-12 respectively.

\subsection{Effects of $\sigma$ on Return Distribution}

The $\sigma$ affects to the kurtosis (peak) of the distribution. When $\sigma$ is zero the volatility is deterministic, because the diffusion process in $d V_{t}$ will be dropped and hence the returns will be normally distributed as in the BSM-model. Increasing $\sigma$ will increase the peak (kurtosis), creating heavy tails on both sides, i.e. the increase in $\sigma$ represents the market volatility is more volatile and higher $\sigma$ shows higher peaks than less one. 


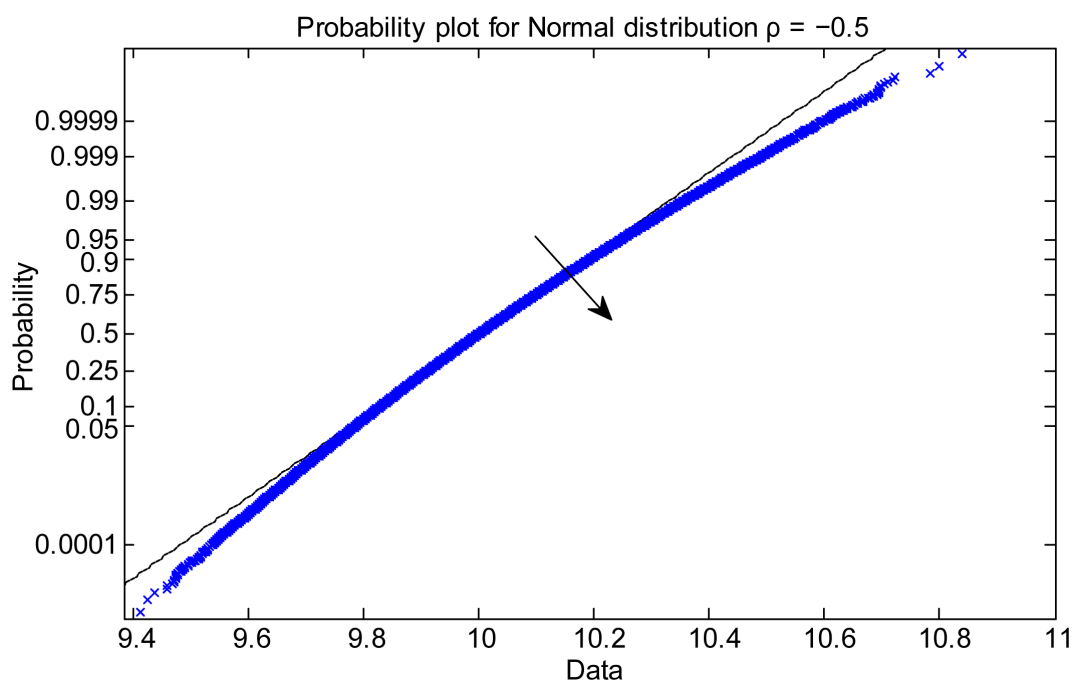

Figure 1. Probability plot, when $\rho$ is smaller than zero asset returns is left skewed.

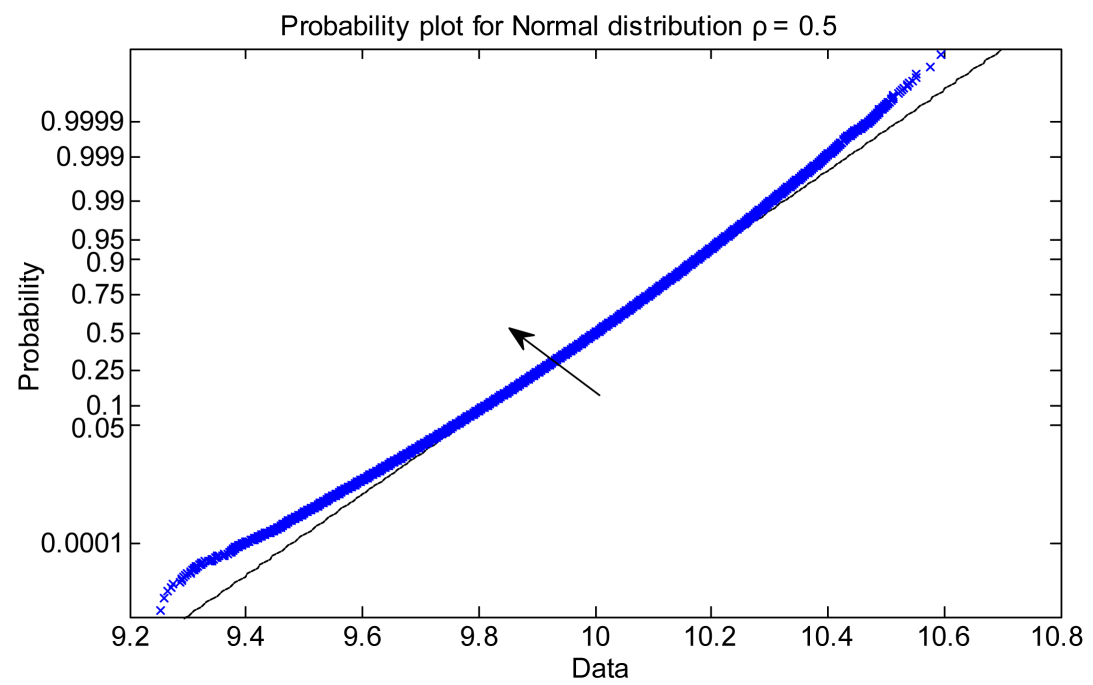

Figure 2. Probability plot, when $\rho$ is greater than zero asset returns is right skewed.



Figure 3. Probability plot, when $\rho$ is equal to zero asset returns is close to zero. 


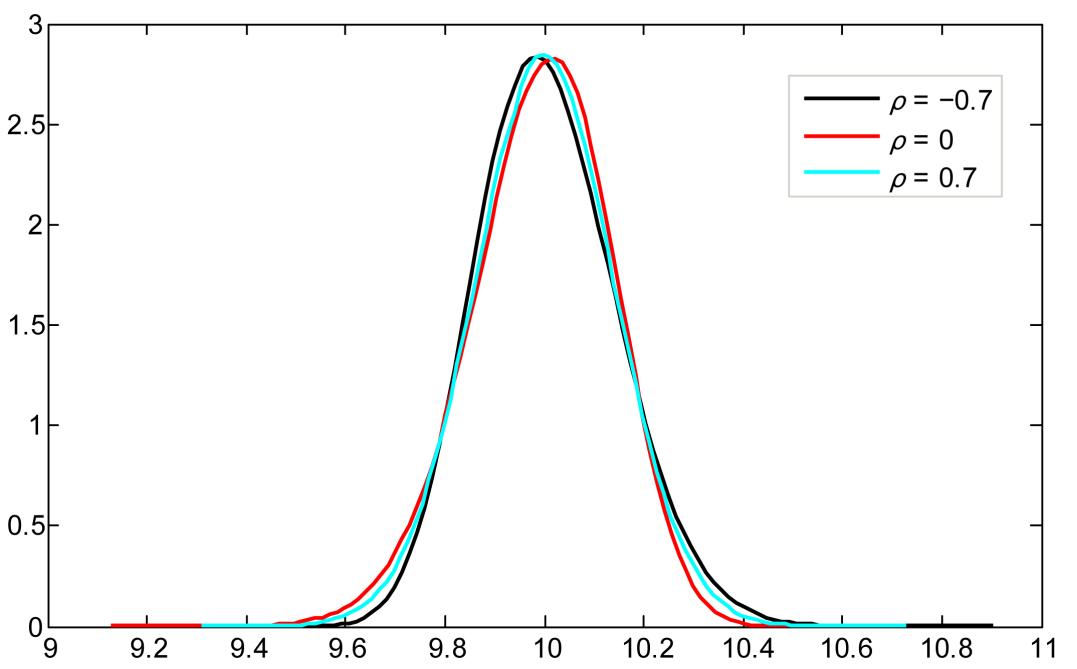

Figure 4. The effect of $\rho$ on the skewness of the density function.

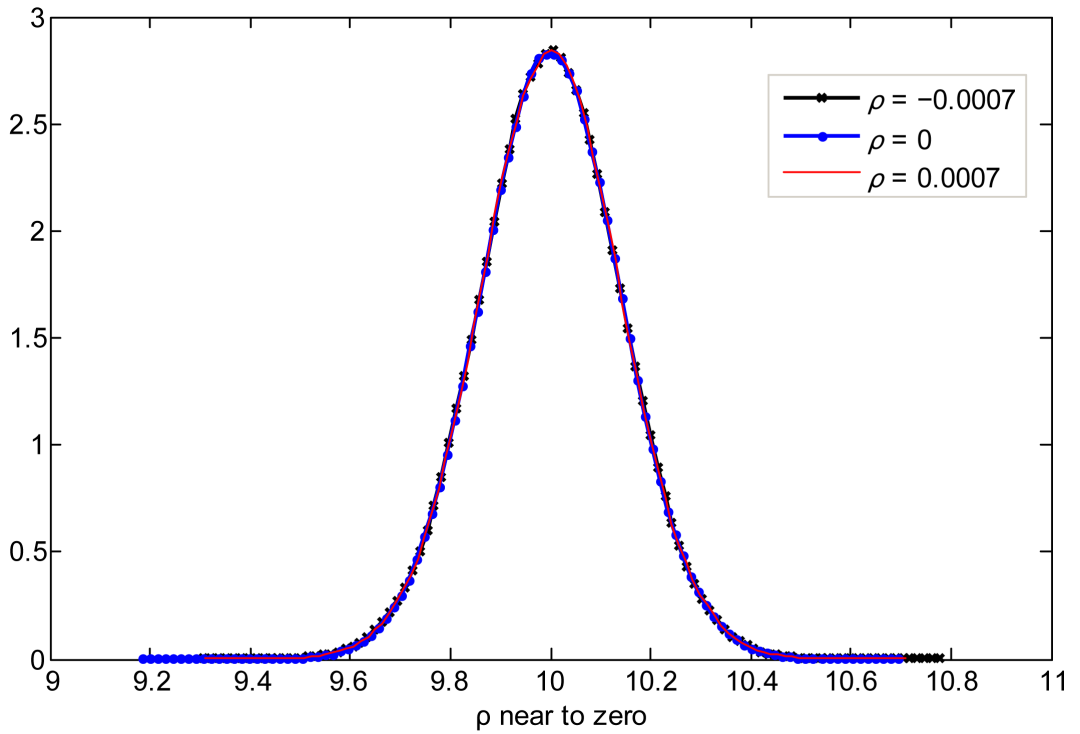

Figure 5. The effect of $\rho$ on the skewness of the density function near to zero.

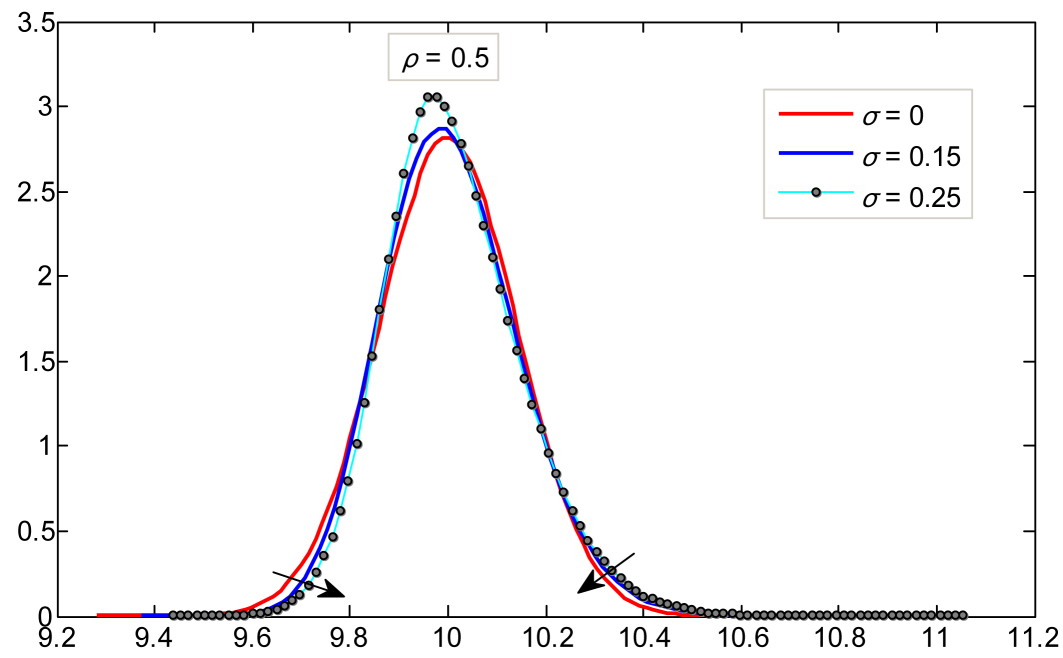

Figure 6. The effect of $\sigma$ on the kurtosis of the density function. 


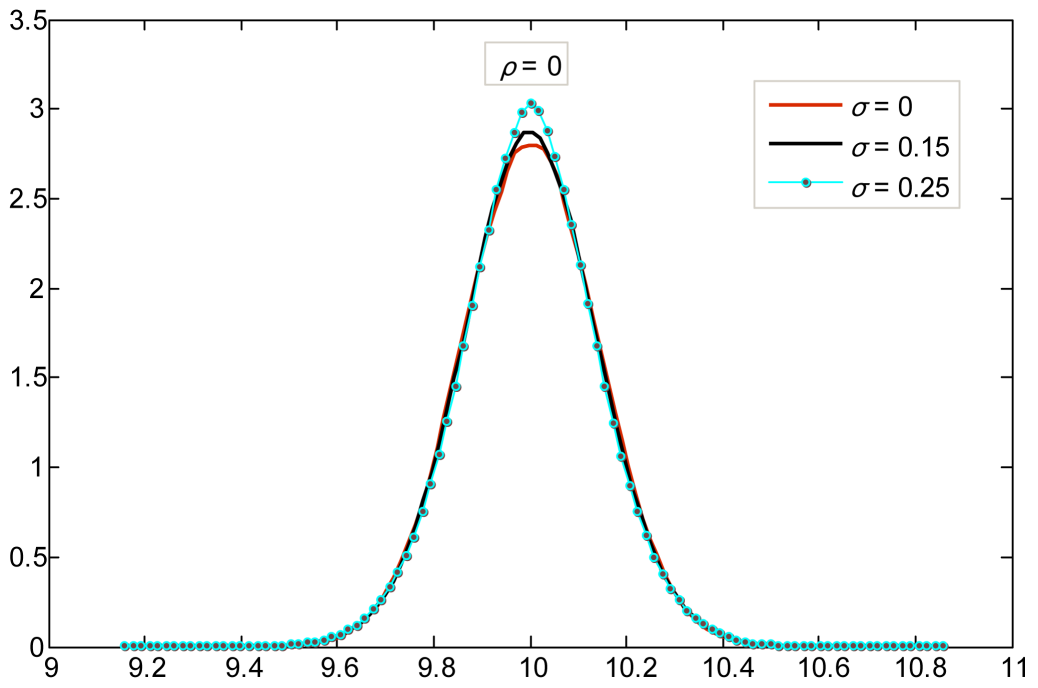

Figure 7. The effect of $\sigma$ on the kurtosis of the density function to zero.

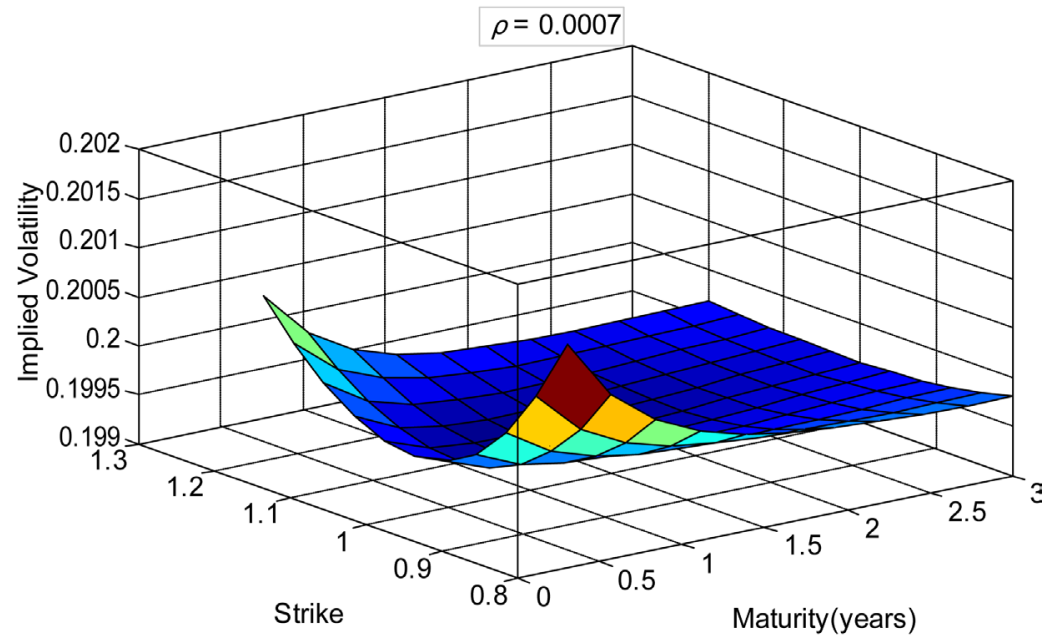

Figure 8. Implied Volatility; $\rho=0.0007 ; \kappa=2 ; \sigma=0.01 ; \theta=0.04 ; v_{0}=0.04 ; r=1 \% ; s=1$ strikes 0.06 - 1.2; maturities: $0.4-3.0$ years.

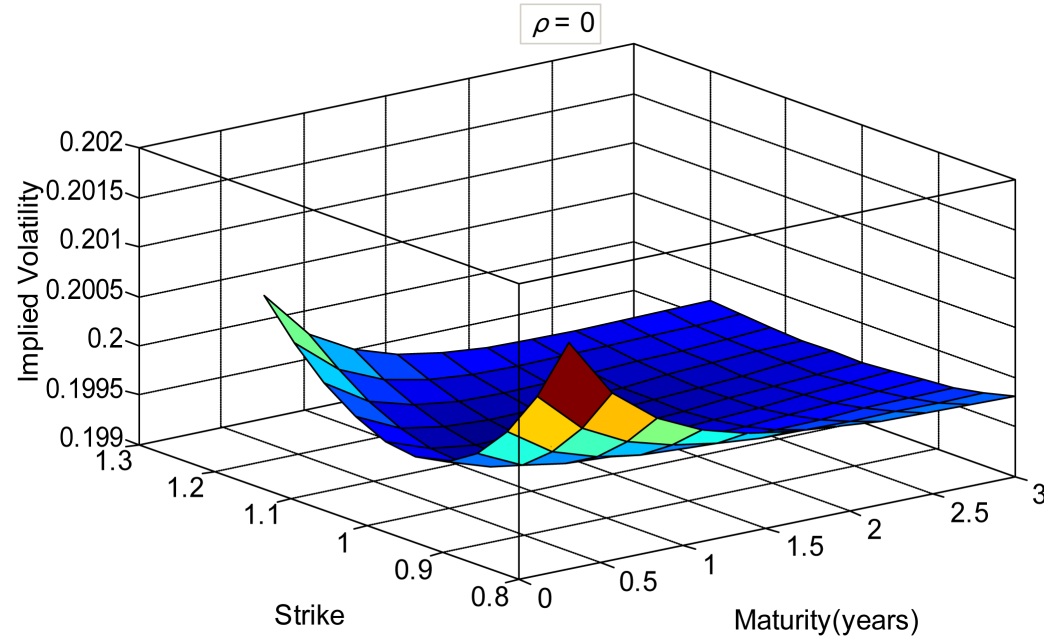

Figure 9. Implied Volatility; $\rho=0 ; \kappa=2 ; \sigma=0.01 ; \theta=0.04 ; V_{0}=0.04 ; r=1 \% ; s=1$ strikes 0.06 - 1.2; maturities: $0.4-3.0$ years. 


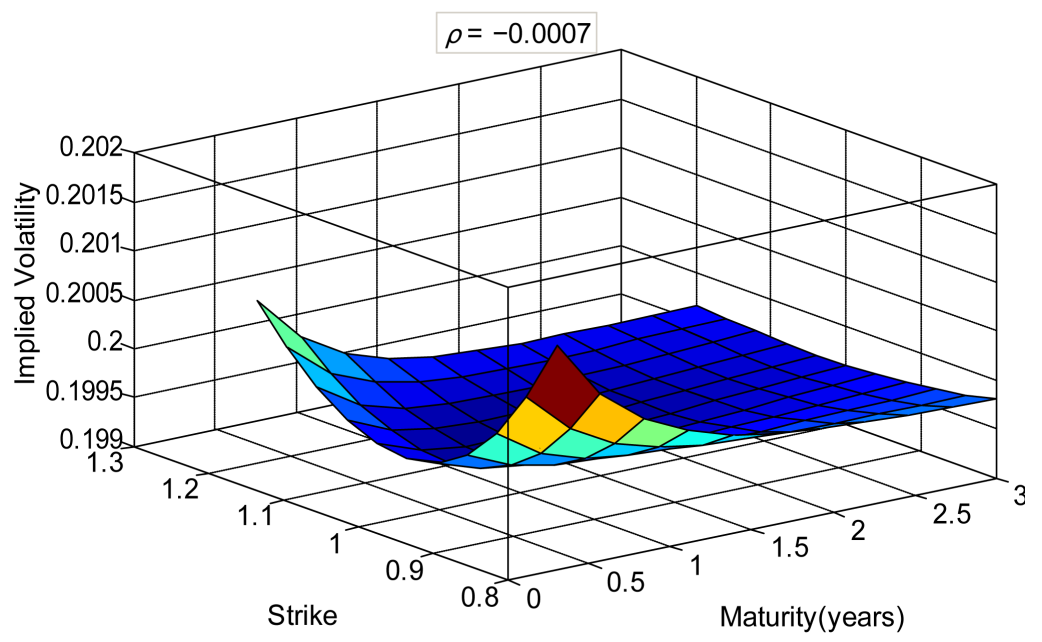

Figure 10. Implied Volatility; $\rho=-0.0007 ; \kappa=2 ; \sigma=0.01 ; \theta=0.04 ; v_{0}=0.04 ; r=1 \% ; s=1$ strikes $0.06-1.2$; maturities: $0.4-3.0$ years.

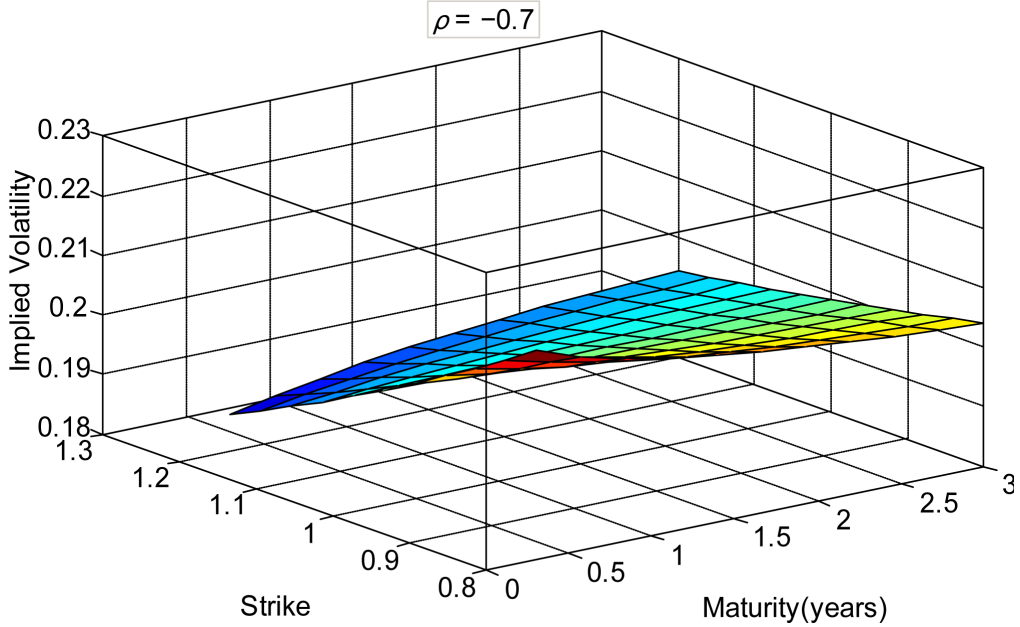

Figure 11. Implied Volatility; $\rho=-0.7 ; \kappa=2 ; \sigma=0.01 ; \theta=0.04 ; v_{0}=0.04 ; r=1 \% ; s=1$ strikes 0.06 - 1.2; maturities: $0.4-3.0$ years.

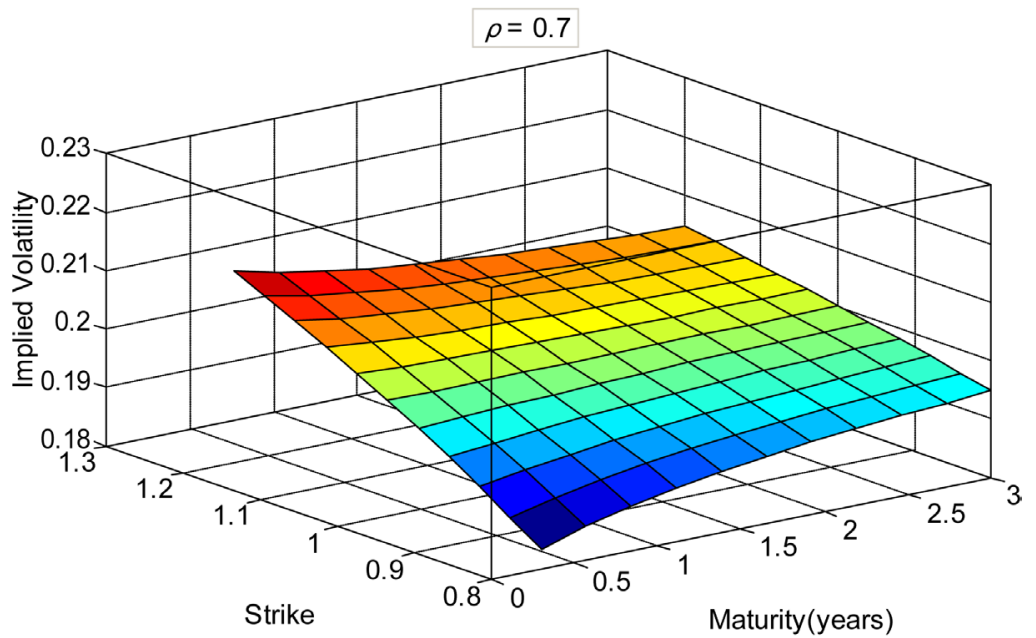

Figure 12. Implied Volatility; $\rho=0.7 ; \kappa=2 ; \sigma=0.01 ; \theta=0.04 ; v_{0}=0.04 ; r=1 \% ; s=1$ strikes $0.06-1.2$; maturities: $0.4-3.0$ years. 
In Figures 8-10, we investigate the effect of $\rho$ on the implied volatility surface generated under Heston's model. Here Maturity (years), Strike and Implied Volatility are denoted by $x$-label, y-label and z-label respectively. In Figure 8 and Figure 9, $\rho=0.007$ and $\rho=0$. Away from the-money options have higher implied volatilities than near-the-money options. This is consistent with the 'smile' shape of implied volatilities in some financial market, e.g. currency options markets. This observation can be explained by the fat-tailed distribution of returns. We can also find that the "smile" flattens. When the time to maturity increases. This is also consistent with the real financial markets. The BS model tends to work well for options with long maturities as a result of the corresponding flattened smile.

In Figure 11, $\rho$ is negative. We can find that in-the-money calls have higher implied volatilities, whereas out-of-the-money calls have lower implied volatilities. This is consistent with the phenomenon of 'volatility skew' in some financial markets, especially the equity options markets.

In Figure 12, $\rho$ is positive which can generate an opposite skew shape compared with Figure 11 such kind of volatility skew may appear in energy options markets.

\section{Conclusions}

Stochastic volatility models tackle one of the most restrictive hypotheses of the Black-Scholes model framework, which assumes that volatility remains constant during the option's life. However, by observing financial markets it becomes apparent that volatility may change dramatically in short time periods and its behavior is clearly not deterministic. Among stochastic volatility models, the Heston model presents two main advantages. First, it models an evolution of the underlying asset which can take into account the asymmetry and excess kurtosis that are typically observed (and expected) in financial asset returns. Second, it provides closed form solutions for the pricing of European options.

The study made in this paper demonstrated a technique for constructing smile and skew consistent prices by violating one of the crude assumptions in the BS model, constant volatility. The result shows that the Heston approximation works really well and only face big problems when options with high time to maturity are to be priced. Another problem is that the approximation gives us incorrect prices when the moneyless is below one. To reduce this problem further studies of the volatility smile could be done and were the skew of options that are not in the money could be compare to options that are in the money and trying to repair this. As one could observe from the results above is that the Heston approximation loses its accuracy as the time to maturity increases, but Black and Scholes is also facing the same type of problem. Since the Heston model was not built on the assumption on non-constant volatility, it showed an improvement of modeling stocks and receiving smile consistent option prices.

\section{Acknowledgements}

The authors would like to express sincere thanks to anonymous reviewers for 
helpful comments on an earlier version of this paper, and they are also grateful to an author assistant of Scientific Research Publishing and an editorial assistant of Journal of Mathematical Finance.

\section{References}

[1] Biswas, M.H.A. (2014) On the Evolution of AIDS/HIV Treatment: An Optimal Control Approach. Current HIV Research, 12, 1-12. https://doi.org/10.2174/1570162X1201140716094638

[2] Hull, J. and John, C. (2008) Options Futures and Other Derivatives. 7th Edition, Pearson Prentice Hall, Upper Saddle River.

[3] Heston, S.L. (1993) A Closed Solution for Options with Stochastic Volatility, with Application to Bond and Currency Options. Review of Financial Studies, 6, 327 343. https://doi.org/10.1093/rfs/6.2.327

[4] Johnson, H. and Shanno, D. (1987) Option Pricing When the Variance Is Changing. Journal of Financial and Quantitative Analysis, 22, 143-151. https://doi.org/10.2307/2330709

[5] Wiggins, J.B. (1987) Option Values under Stochastic Volatility: Theory and Empirical Estimates. Journal of Financial Economics, 19, 351-372.

[6] Hull, J. and White, A. (1988) An Analysis of the Bias in Option Pricing Caused by a Stochastic Volatility. Advances in Futures and Options Research, 3, 27-61.

[7] Hull, J. and White, A. (1987) The Pricing of Options on Assets with Stochastic Volatilities. Journal of Finance, 42, 281-300. https://doi.org/10.1111/j.1540-6261.1987.tb02568.x

[8] Scott, L.O. (1987) Option Pricing When the Variance Changes Randomly: Theory, Estimation and an Application. Journal of Financial and Quantitative Analysis, 22, 419-438. https://doi.org/10.2307/2330793

[9] Stein, E. and Stein, C.J. (1991) Stock Priced Distributions with Stochastic Volatility: An Analytical Approach. Review of Financial Studies, 4, 727-752. https://doi.org/10.1093/rfs/4.4.727

[10] Biswas, M.H.A., Rahman, T. and Haque, N. (2016) Modeling the Potential Impacts of Global Climate Change in Bangladesh: An Optimal Control Approach. Journal of Fundamental Applied Science, 8, 1-19. https://doi.org/10.4314/jfas.v8i1.1

[11] Mondal, M.K. and Biswas, M.H.A. (2015) Modeling the Transmission Dynamics of the Nipah Virus Infection. Proceedings of 1 st International Conference on Mathematics and It's Applications, Khulna, 23 December 2015, 22-26.

[12] Neilan, R.M. and Lenhart, S. (2010) An Introduction to Optimal Control with an Application in Disease Modeling. DIMACS Series in Discrete Mathematics, 75, 6781. https://doi.org/10.1090/dimacs/075/03

[13] Biswas, M.H.A. and Haque, M.M. (2016) Nonlinear Dynamical Systems in Modeling and Control of Infectious Diseases. In: Pinelas, S., Došlá, Z., Došlý, O. and Kloeden, P., Eds., Differential and Difference Equations with Applications, Springer Proceedings in Mathematics \& Statistics, Vol. 164, Springer, Cham., 149-158. https://doi.org/10.1007/978-3-319-32857-7_15

[14] Berezansky, L., Idels, L. and Kipnis, M. (2011) Mathematical Model of Marine Protected Areas. IMA Journal of Applied Mathematics, 76, 312-325. https://doi.org/10.1093/imamat/hxq043

[15] Biswas, M.H.A. (2014) Optimal Control of Nipah Virus (NiV) Infections: A Bangladesh Scenario. Journal of Pure and Applied Mathematics. Advances and Appli- 
cations, 12, 77-104.

[16] Murray, J.D. (1989) Mathematical Biology: Biomathematics. 2nd Edition, Springer, New York. https://doi.org/10.1007/978-3-662-08539-4

[17] Black, F. and Scholes, M. (1973) Valuation of Options and Corporate Liabilities. Journal of Political Economy, 81, 637-654. https://doi.org/10.1086/260062

[18] Moodley, N. (2005) The Heston Model: A Practical Approach with Matlab Code. B.Sc. Thesis, University of the Witwatersrand, Johannesburg.

Submit or recommend next manuscript to SCIRP and we will provide best service for you:

Accepting pre-submission inquiries through Email, Facebook, LinkedIn, Twitter, etc. A wide selection of journals (inclusive of 9 subjects, more than 200 journals)

Providing 24-hour high-quality service

User-friendly online submission system

Fair and swift peer-review system

Efficient typesetting and proofreading procedure

Display of the result of downloads and visits, as well as the number of cited articles Maximum dissemination of your research work

Submit your manuscript at: http://papersubmission.scirp.org/

Or contact jmf@scirp.org 